\title{
The Influence of Removing the Perception of Achievement on Performance in Volitional
} Time-to-Exhaustion Cycle Ergometer Trials: A Brief Review

Ross Lorimer and John Babraj

Division of Sport and Exercise Sciences, University of Abertay Dundee, Dundee, United Kingdom

\section{ABSTRACT}

The purpose of this brief report is to report the findings of an investigation of the potential influence of the removal of achievement feedback on subjects undertaking maximal fitness testing. Nine subjects were asked to complete two incremental volitional time-to-exhaustion cycle ergometer trials, one with feedback and one without. The final minute of each trial, following the last increase in increment in resistance was divided into four 15-second intervals. A frequency count made of how many subjects stopped when within each interval. A paired samples t-test showed no significant difference $(p>0.05)$ between the mean total time-to-exhaustion of the blinded and feedback conditions. A chi-square test was used to determine that there was a significant difference between the expected frequencies and the observed frequencies in the feedback condition. With feedback, subjects were significantly more likely to stop in the first interval (015s) while without feedback subjects were evenly distributed across all four intervals. There was also a non-significant, but medium-to-large difference, in time-toexhaustion with those in the feedback condition going an average 39.44s longer. This suggests a potential psychological element related to goal achievement that influences performance in incremental volitional time-to-exhaustion cycle ergometer trials. The information subjects have available on which to base goals (level of increment, time etc.) needs to be managed to prevent spontaneous goal setting and ensure true time-to-exhaustion is achieved.

Key Words: Goal-setting, motivation, fitness testing 


\section{INTRODUCTION}

Volitional time-to-exhaustion is a common tool in strength and conditioning and involves subjects undertaking a physical test (e.g., cycling) until a subject expresses an inability to continue exercising (5). This is a brief report following on from the work of Babraj and Lorimer (5). They found that subjects completing a volitional time-toexhaustion on a cycle ergometer were significantly more likely to end their trial near to an increase in increment of resistance or another indicator of achievement such as whole minutes completed.

During a volitional time-to-exhaustion cycle ergometer trial subjects are not provided with any goal except to continue cycling until they are no longer able to maintain the required work level such as a predetermined rpm (1). Under these conditions, subjects are essentially asked to 'do their best' and continue the trial until volitional exhaustion. The aim of this type of fitness assessment is to have subjects continue working until they become metabolically exhausted in order to estimate their aerobic fitness as an indicator of performance, health or a successful training intervention (1). During these trials, subjects frequently have access to a range of achievement-related feedback such as total time, rpm, and the incremental increase in resistance at each level. While it is recommended that researchers and practitioners withhold total time from subjects, those subjects can still use the incremental increase in resistance as an indicator of time completed. Previous work has shown that subjects use this information to set goals (5) and similar patterns have been observed in studies using these types of trials as a fitness assessment (e.g., 2). This leads to subjects being unwilling to continue upon either perceiving the achievement of their goal or when unable to perceive the possibility of achieving another harder goal such as the next increase in increment of resistance (5).

The underpinning argument of goal setting theory is that subject performance is mediated by the subject's conscious goals and the achievement of these (3). It has previously been shown that subjects will spontaneously set goals in the context of physical activity (4). It has also been shown that these spontaneously set goals appear to limit performance in volitional time-toexhaustion cycle ergometer trials (5). What is not known is if the removal of achievement related feedback would counter this process. The aim of this study was to examine the influence of perception of achievement on performance in volitional time-toexhaustion cycle ergometer trials. It was hypothesised that subjects would be significantly more likely to stop immediately following an increase in the increment of resistance on an incremental volitional time-to-exhaustion, and when subjects had no access to achievementrelated feedback there would be no discernible pattern to their stopping point in relation increases in the increment of resistance.

\section{METHODS}

\section{Subjects}

A convenience sample of recreationally active males was recruited ( $N=9 ; 26 \pm 8 \mathrm{y}$; $81 \pm 7 \mathrm{~kg} ; 1.82 \pm 0.06 \mathrm{~m}$ ). All subjects were invited to participate in the study. After explanation of the aims and background of 
the study the subjects were asked to complete a physical activity readiness questionnaire and an informed consent form. The study was carried out in line with the declaration of Helsinki and consent was gained from the Institutional Ethics Committee before any testing was undertaken.

\section{Procedures}

Subjects completed two time-toexhaustion cycle ergometer trials, with either feedback or blinded, in a random order. In the feedback condition, subjects had access to achievement-related feedback - they were able to see to total time, rpm, and the incremental increase in resistance at each level. In the blinded condition, subjects were blindfolded and had no access to achievement-related feedback. For each subject the first trial was arranged for a mutually convenient time. The second trial was then held at the same time of day following a minimum of 48-hours.

All trials consisted of a standard incremental time to exhaustion test (1) carried out on a Monarch cycle ergometer (model 894E). Subjects first completed a 4-minute warm-up period at a speed greater than 60rpm against the resistance of the unweighted cradle. After these 4 minutes, and after every minute thereafter, an increment of $0.5 \mathrm{~kg}$ was placed on the bike cradle using calibrated weight plates. Load was standardised rather than normalised for body mass as this is most frequently normal practice. However, as analysis was a within subjects study design this choice would not influence the results. Increase in increments continued every minute until the subjects indicated their desire to stop or were judged to no longer be maintain a minimum 60-rpm; this was decided when a subject's speed fell below 60-rpm for more than 10 s despite a verbal warning to increase their speed. Total time to exhaustion was recorded, as was time since the last increment of resistance had been added.

\section{Statistical Analyses}

A Shapiro-Wilks test was run to confirm normality and then a paired samples t-test was carried out to examine the observed difference between the mean total timeto-exhaustion of the blinded and feedback conditions. Following this a chi-square test was used to determine whether there was a significant difference between the time since the last increment of resistance, by separating the potential $60 \mathrm{~s}$ into four $15 \mathrm{~s}$ sections and comparing the expected frequencies (stopping times appear randomly distributed across the four 15second intervals) and the observed frequencies (distribution of stopping times across four 15-second intervals). As there was an expected frequency count of less than five in each interval a Yates correction was used (6). A p-value of 0.05 or less was determined to be significant.

\section{RESULTS}

No significant effect was found between the mean total time-to-exhaustion of the blinded (626 $\pm 74 \mathrm{~s})$ and feedback (665 \pm 68s) conditions $(t(8)=-1.72, \quad p=0.123)$. However, there was a notable absolute difference between the two conditions (39s longer in feedback condition) with a medium-to-large effect size $(d=0.66)$. Figure 1. shows the observed frequencies for both cycle ergometer conditions in each of the four 15-second intervals of the 
final minute. For the blinded condition there was no significant difference, 国 2 ( 3 , $\mathrm{N}=9)=2.11, \mathrm{p}=0.55$. For the feedback condition there was a significant difference between the expected frequencies and the

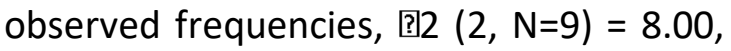
$\mathrm{p}=0.02$, with the majority of subjects stopping in the first interval (0-15s) and none within the last interval (45-60s).

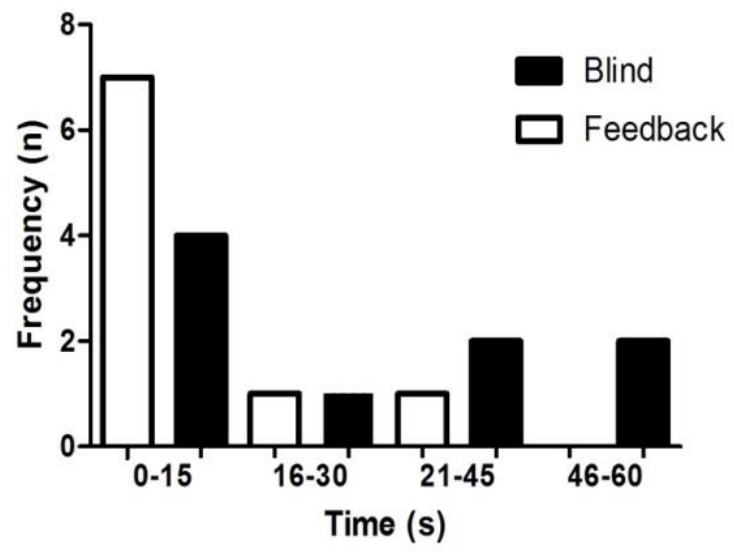

Figure 1. Observed frequencies of stopping points over the minute following the final increment of resistance

\section{DISCUSSION}

The aim of this study was to examine the influence of perception of achievement on performance in volitional time-toexhaustion cycle ergometer trials. This was done by comparing conditions where participants did and did not have access to information on which to judge their performance. Subjects in the feedback condition were significantly more likely to stop within 15 s following an increase in the increment of resistance than at any point in the following 45s. In contrast, when undertaking the blinded condition, subjects showed no discernible pattern to their stopping point in relation to increases in the increment of resistance. This pattern of findings suggests that in the feedback condition, subjects were stopping when they perceived they had reached the end of a stage but would be unlikely to complete the next stage (5). This means they may not have been continuing their cycle ergometer trial until complete metabolic exhaustion. Additionally, while there was no significant difference in the mean total time-toexhaustion, subjects went for an average of 39s longer in the feedback trials. The variation in stopping point represents a potential threat to the validity of volitional time-to-exhaustion cycle ergometer trials (5); however, the difference in total timeto-exhaustion suggests that managing this is not as straightforward as removing achievement-related feedback.

The pattern observed in the feedback trials show that subjects typically end their trial almost immediately following an increment of resistance and never stopped their trial immediately before an increment of resistance. It can be suggested that subjects were using the achievement-related feedback available (increments of resistance placed on the cradle) to determine how well they were doing $(5,3)$. Subjects appeared to use this feedback to set spontaneous goals based on achieving a final increase in increment of resistance before failure (3). This is in line with previous studies that have found that when examining the effect of goal setting on performance, subjects without goals will spontaneously set them $(3,4,5)$. The fact there was no discernable pattern in the blinded condition means that it is unlikely subjects ended their trial in relation to increases in increment of resistance. This may suggest that in the absence of achievement-related feedback the subjects are more likely to continue until volitional exhaustion. 
What is not clear is if the use of achievement-related feedback is improving or impairing a subjects' performance. Locke (3) has argued that spontaneously set goals can improve the performance of subjects in context of physical activity while Lorimer and Babraj (5) have suggested it may hinder performance in time-to-exhaustion trials. Subjects in the feedback trials stopped almost immediately on reaching an increment in resistance, representing a spontaneously set goal, which suggests a limited performance. Conversely, it has been suggested that subjects near to exhaustion will continue a trial in an effort to reach the next increment of resistance as long as they perceive it to be close (5). This second supposition is weakly supported by the medium-to-large, but non-significant, difference total time-toexhaustion, with subjects going for longer in the feedback condition. However, a counter argument is that in the blinded condition, subjects had no access to feedback regarding rpm, and anecdotally, subjects in the blinded trials cycled at a higher rate and may have reached exhaustion more quickly than in the feedback trials. This needs further investigation.

\section{CONCLUSION}

The purpose of this brief report was to highlight the potential influence of perception of achievement, related to spontaneously set goals, on performance in volitional time-to-exhaustion trials. Subjects appear to use the achievementrelated feedback when it is available (either internally or externally) to determine how well they are doing. Subjects use this feedback to set specific goals based on achieving a final increase in increment of resistance before failure. When the feedback is removed, subjects are unable to set specific goals and display no discernable pattern in their stopping point. Future investigations need to continue expand on this area of research to find an appropriate balance of achievement-related feedback that restricts spontaneously set goals but that allows subjects to pace themselves appropriately. 


\section{References}

1. Basset, F, and Boulay, M. Treadmill and cycle ergometer tests are interchangeable to monitor triathletes annual training. Journal of Sports Science and Medicine 2: 110-116, 2003.

https://www.ncbi.nlm.nih.gov/pmc/articles/ PMC3942637/

2. Jakeman, J, Adamson, S, \& Babraj, J. (2012). Extremely short duration high-intensity training substantially improves endurance performance in triathletes. Applied Physiology, Nutrition, and Metabolism, 37, 976-981, 2012.

https://www.nrcresearchpress.com/doi/abs/ 10.1139/h2012-083\#.XqlHFv9Kg2w

3. Locke, E. Problems with goal-setting research in sports - and their solution. Journal of Sport and Exercise Psychology 13: 311-316, 1991. https://journals.humankinetics.com/view/jo urnals/jsep/13/3/article-p311.xml

4. Locke, EA, and Latham, GP. Building a practically useful theory of goal and task motivation. American Psychologist, 57, 705717, 2002.

https://med.stanford.edu/content/dam/sm/ s-spire/documents/PD.locke-and-lathamretrospective Paper.pdf

5. Lorimer, R, and Babraj, J. The potential influence of perception of achievement on performance in volitional time-to-exhaustion cycle ergometer trials. Journal of Strength and Conditioning Research 27: 1546-1548, 2013.

https://www.ncbi.nlm.nih.gov/pubmed/230 $\underline{37613}$

6. Rhoades, $\mathrm{H}$, and Overall, J. A sample size correction for Pearson chi-square in $2 \times 2$ contingency tables. Psychological Bulletin, 9, 418, 1982.

https://psycnet.apa.org/record/1982-11179$\underline{001}$ 